\title{
Nódulo tiroideo frío. Características clínicas y anatomo- patológicas en pacientes migrantes de zonas con deficiencia de yodo y de la costa.
}

Cold thyroid nodule. Clinical and anatomopathological characteristics in migrant patients from iodine deficiency areas and from the coast.

VILLENA Jaime ${ }^{1}$, FERRUFINO Juan $C^{2}$ y KLINGE Germán ${ }^{3}$.

${ }^{1}$ Profesor Auxiliar de Medicina. Universidad Peruana Cayetano Heredia, Servicio de Endocrinología. Hospital Nacional Cayetano Heredia.

${ }^{2}$ Profesor Auxiliar de Patología UPCH. Servicio de Patología, Hospital Nacional Cayetano Heredia.

${ }^{3}$ Servicio de Patología, Hospital Nacional Cayetano Heredia

\section{SUMMARY}

The clinical and pathological charactheristics of 237 patients out 1468 patients with thyroid diseases, who had cold thyroid nodules (CTN), were studied retrospectively, 133 of them were inmigrants from iodine deficiency areas (IDA) from the Andean region in Perú, and 104 from the coast, an iodine sufficent area (ISA). CTN comprised $50.5 \%$ of nodular goiters in ISA and only $29.8 \%$ in IDA $\left(p=3 * 10^{7}\right)$. Patients from IDA were older than those from ISA, $46.2 \pm 13.4$ years vs $35.5 \pm 14.4$ ( $p=0.0001)$. Cystic lesions were found in $\mathbf{4 . 6 \%}$ of nodules, without difference between groups. Graves disease was present in $3.8 \%$ of patients from IDA and in $0.9 \%$ from ISA $(p=0.23)$. Fifty seven patients from ISA and 55 from IDA were operated on. Cáncer was found in $25 \%$ of those from IDA and in $32 \%$ from ISA $(\mathrm{P}=0.69)$. Papillary cancer was the commonest variant in both groups. The more frequent bening lesions were colloid nodular goiter $(46.2 \%)$ and follicular adenomas $(31.2 \%)$, without difference between groups. (Rev Med Hered 1993; 4(4): 188-193)

KEY WORDS: Thyroid nodule, cold thyroid nodule, nodular goiter, endemic goiter, iodine deficiency.

\section{RESUMEN}

Se han estudiado retrospectivamente 237 pacientes con nódulo tiroideo frió (NTF), 133 inmigrantes de zonas con deficiencia de yodo (ZDY) y 104 de la costa (donde no hay deficiencia de yodo), de un total de 1468 pacientes con patología tiroidea, atendidos. El 
NTF representó el $50.5 \%$ del total de bocios nodulares en la costa y $29.8 \%$ en ZDY $\left(\mathrm{p}=3 * 10^{-7}\right)$. La edad promedio de los pacientes de ZDY fue mayor $46.2 \pm 13.4$ años vs. $35.5 \pm 14.4(\mathrm{p}=0.0001)$. El $4.6 \%$ de los nódulos fueron quísticos sin diferencia entre ambos grupos. El $2.5 \%$ tenían Enfermedad de Graves concomitante, 3.8\% en ZDY y $0.9 \%$ en la costa $(\mathrm{p}=0.23)$. Fueron operados 112 pacientes, 57 de la costa y 55 de ZDY. Se encontró cáncer en $25 \%$ de ZDY y $32 \%$ de la costa $(\mathrm{p}=0.69)$, siendo el papilar el cáncer más frecuente en ambos grupos. Las lesiones benignas más frecuentes fueron el bocio nodular coloide (46.2\%) y el adenoma folicular (31.2\%), sin diferencia entre ambos grupos estudiados. (Rev Med Hered 1993; 4(4): 188-193)

PALABRAS CLAVE: Nódulo tiroideo, nódulo tiroideo frío, bocio nodular, bocio endémico, deficiencia de yodo.

\section{INTRODUCCIÓN}

El nódulo tiroideo (NT), es una afección frecuente, detectable clínicamente en 4.2\% a 5.3\% de la población $(1,2)$, siendo su prevalencia aún mayor en zonas con deficiencia de yodo (ZDY) $(3,4)$.

Puede producir síntomas compresivos, tirotoxicosis o albergar un cáncer. La probabilidad de cáncer tiroideo, considerado una neoplasia letal (5), es mayor cuando el nódulo es sólido y frío a la gammagrafía (6), de ahí que numerosas revisiones al respecto aparecen periódicamente en la literatura, para el mejor manejo de esta patología (6-16).

En ZDY se ha descrito una mayor frecuencia de cáncer tiroideo, particularmente del tipo folicular y anaplásico (17-20), sin embargo en los casos de NT frío, una proporción similar e incluso menor, sobretodo en mujeres, ha sido hallada recientemente (21).

En el Perú, aún subsiste la defíciencia de yodo, en varias regiones (22), con una gran migración hacia Lima, parte de la cual es captada por nuestro Hospital.

Con el objeto de contribuir al conocimiento de las características clínicas y anatopatológicas de los pacientes portadores de NT frío en nuestro medio y la influencia que pudiera tener la deficiencia previa de yodo en esta patología, es que se presenta la experiencia acumulada en 15 años, en el Servicio de Endocrinología.

Una cuarta parte de la casuística ha sido presentada anteriormente (23) y aspectos generales han sido publicados recientemente (4).

\section{MATERIAL Y MÉTODO}

Se han revisado en forma retrospectiva las historias clínicas de 1,468 pacientes con patología tiroidea atendidos en el Servicio de Endocrinología del Hospital Nacional Cayetano Heredia de 1970 a 1985, a quienes se clasificó de acuerdo a su lugar de procedencia en dos grupos: uno compuesto por 811 (55.2\%) pacientes oriundos y procedentes de la costa, donde no hay deficiencia de yodo y otro por $657(44.8 \%)$ casos que 
procedían de las provincias de la sierra de los departamentos de Lima, Ancash, La Libertad, Ayacucho, Amazonas y de algunos villorrios de la selva, donde aún existe deficiencia de yodo (ZDY), con prevalencia de bocio en edad escolar de $24 \%$ a $38.9 \%$ (22).

Por palpación se clasificó los bocios en difusos (BD), uninodulares (BUN) o multinodulares $(\mathrm{BMN})$.

Se hizo el diagnóstico de nódulo tiroideo frío (NTF) en 237 casos de bocio uninodular, que presentaban hipocaptación del radioyodo en la gammagrafía (24). Ciento cuatro (43.9\%) fueron de la costa y 133 (56.1\%) de ZDY.

Ciento doce (47.3\%) pacientes fueron operados. Cincuenta y siete $(54.8 \%)$ de la costa y 55 $(41.4 \%)$ de ZDY. Las indicaciones quirúrgicas fueron hechas por el médico tratante, por tener el paciente factores de riesgo por cáncer tiroideo, como son, dureza, edad joven o avanzada, y adenopatía satélite. Otras veces por gran tamaño, y desde 1982 por tener una biopsia por Aspiración (ABC) compatible con cáncer.

Todos los cortes histológicos fueron revisados por dos de nosotros (JCF, GK) y clasificados según criterios de Sommers (25).

La función tiroidea fue estimada por la determinación del PBI, $\mathrm{T}_{3}$ ó $\mathrm{T}_{4}$. Los pacientes fueron catalogados como hipertiroideos, hipotiroideos o eutiroideos según criterio clínico del examinador y por tener pruebas de función tiroidea compatibles.

La gammagrafía tiroidea fue realizada con $\mathrm{I}^{131}$ en un gammagrafo rectilíneo en el Servicio de Medicina Nuclear de la Universidad Peruana Cayetano Heredia.

EL PBI fue determinado por el método de Benotti (26), el $\mathrm{T}_{4}$ inicialmente por unión proteica competitiva (27) y desde 1978 por radioinmunoensayo (DPC, Los Angeles, CA), al igual que el $\mathrm{T}_{3}$.

Los datos son expresados en promedio \pm desviación estándar. El análisis estadístico fue hecho mediante el cálculo de Z, para muestras grandes y por la prueba t de Student y Chicuadrado, para muestras pequeñas y proporciones, respectivamente.

\section{RESULTADOS}

El NTF representó el $20.2 \%$ de la patología tiroidea total entre procedentes de ZDY y $12.8 \%$ entre los de la costa ( $\mathrm{p}=0.00012)$. Constituyó el $29.8 \%$ de los bocios nodulares en el primer grupo y $50.5 \%$ en el segundo $(\mathrm{p}=0000003)$ y correspondió al $51.8 \%$ de los nódulos tiroideos únicos en los de ZDY y $63.8 \%$ en los de la costa $(\mathrm{p}=0.015)$.

La edad promedio de los pacientes de ZDY fue mayor, $46.2 \pm 13.4$ años vs. $35.5 \pm 14.4$ $(\mathrm{p}<0.0001)$. La mayor frecuencia se observó entre los 41 a 50 años en los pacientes de ZYD y entre los 31 a 40, en los de la costa (Figura $\mathrm{N}^{\circ} 1$ ). 


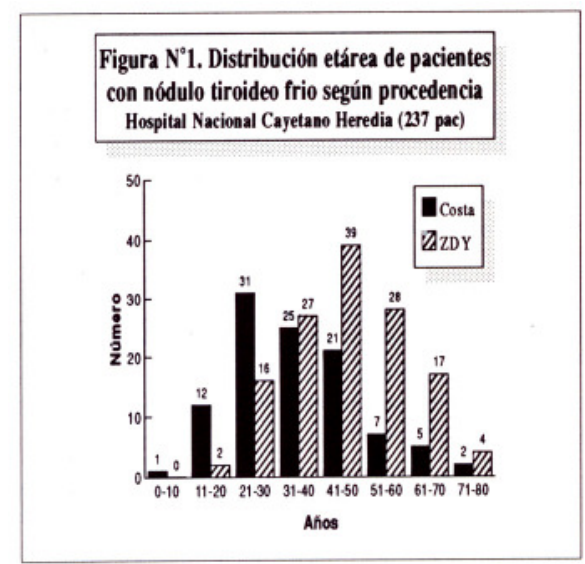

La programación de mujeres fue de $95.5 \%$ en el primer grupo y $91.1 \%$ en el segundo $(\mathrm{p}=0.302)$.

El 4.6\% de los nódulos fueron quísticos, a juzgar por la $\mathrm{ABC}$ ó los hallazgos quirúrgicos, $5.3 \%$ en procedentes de ZDY y $3.8 \%$ en los de la costa $(p=0.76)$.

En el 97.5\% de los casos los pacientes fueron eutiroideos, 2.5\% tenían Enfermedad de Graves concomitante, $3.8 \%$ en los de ZDY y $0.96 \%$ en los de la costa $(p=0.23)$.

De los 112 pacientes operados, en 29\% se encontró cáncer, siendo el más frecuente el papilar seguido del mixto folicular. No hubo diferencia en el porcentaje ni tipos de cáncer entre procedentes de ZDY y de la costa, $25 \%$ vs $32 \%$, respectivamente $(p=0.69)$ (Tabla $\mathrm{N}^{\circ} 1$ ).

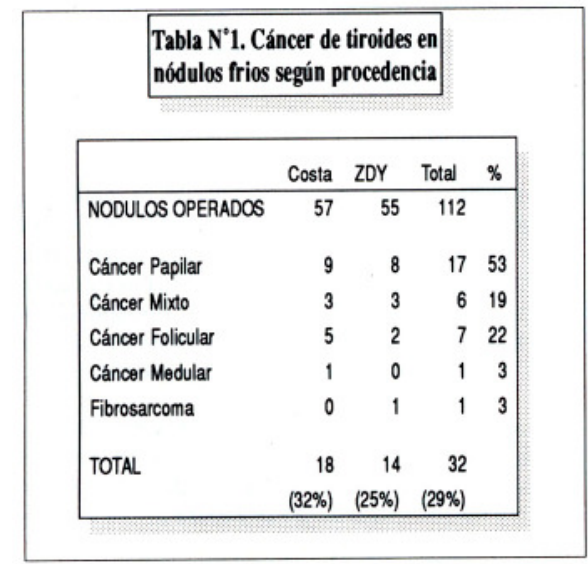

Con respecto al total de NTF, se encontró cáncer en $13.5 \%(32 / 237)$ del total, $10.5 \%$ $(14 / 133)$ en los de zonas endémicas y $17.3 \%$ (18/104) en los de la costa $(p=0.2)$. En $71 \%$ se encontró lesiones benignas, siendo las más frecuentes el bocio nodular coloide y el adenoma folicular. No hubo difrencia entre el grupo de ZDY y de la costa, 75\% VS 68\%, respectivamente $(\mathrm{p}=0.274)\left(\right.$ Tabla $\left.\mathrm{N}^{\circ} 2\right)$. 


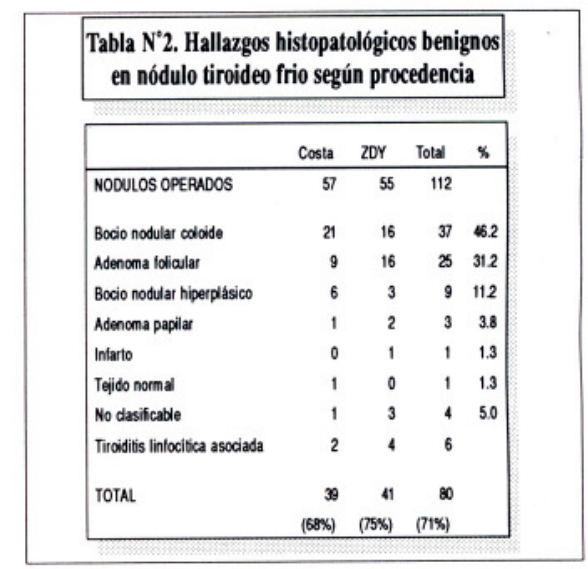

\section{DISCUSIÓN}

El NTF representó el $20 \%$ de la patología tiroidea total en pacientes de ZDY y sólo el $12.8 \%$ en la costa, esto debido a la mayor frecuencia de bocios nodulares en ZDY $(3,4)$, mientras que en la costa el bocio difuso es más prevalerte.

Por otra parte el NTF representa el $29.8 \%$ de los bocios nodulares en ZDY y $50.5 \%$ en la costa, explicable por la mayor frecuencia de bocios multinodulares en ZDY, que en nuestro servicio representan el $42.6 \%$ de los bocios nodulares en ZDY y sólo el $20 \%$ en la costa (4).

El menor porcentaje de NTF con respecto al total de bocios uninodulares que en la costa $(51.8 \%$ vs. $63.8 \%)$, se debe a la mayor frecuencia de nódulos calientes en regiones con deficiencia de yodo (28.29). Así, en nuestro hospital, en procedentes de ZDY, el nódulo caliente constituye el $35.3 \%$ de los BUN vs. $21.1 \%$ en los de Lima (4).

La edad de nuestros pacientes de ZDY fue mayor que los de Lima. En el único estudio comparativo similar al nuestro, encontrado en la literatura, Belfiore y col (21) en Catania, Italia, no encuentran diferencia en la edad de presentación en ambas regiones. Dada las diferencias socioeconómicas, es probable que nuestros pacientes acudan tardíamente a los Servicios de Salud, por menor accesibilidad o por considerar muchas veces el bocio como algo "natural".

Hallamos un $4.6 \%$ de lesiones quísticos, sin diferencia en los grupos estudiados. Creemos que esta proporción está subestimada, ya que no todos los nódulos fueron punzados u operados y a ningún paciente se le realizó ecografía, que es un método sensible para detectarlas (15).

Sin embargo en el análisis de casos de NTF operados hasta 1975 en nuestro hospital, Cipriano (23) no reporta ningún caso en 42 pacientes operados.

En la literatura del $6 \%$ al $32 \%$ de los nódulos solitarios son quísticos $(6,7,13,15,16,30$, 31) representando generalmente hemorragias en adenoma folicular, o necrosis de cáncer papilar, siendo las lesiones quísticas puras, raras $(7,30)$. 
El porcentaje de malignidad en lesiones quísticas varía entre $7 \%$ al $14 \%(6,30)$, habiéndose sugerido desterrar el concepto que los quistes tiroideos son usualmente benignos (30). En el manejo de éstos la $\mathrm{ABC}$ es fundamental, ya que además de dar información sobre la posibilidad de cáncer, los quistes remiten en un $50 \%$ después de la punción, como fue nuestra experiencia. Para los quistes recurrentes se ha sugerido la cirugía (30) y la inyección de tetraciclina $(31,32)$.

En el $71 \%$ de los casos operados, las lesiones encontradas fueron benignas, siendo la más frecuente el bocio nodular coloide, seguida del adenoma folicular, sin diferencia significativa entre ambos grupos estudiados. Estos resultados concuerdan con lo hallado por Cipriano previamente (23).

En la revisión de la literatura del $42 \%$ al $77 \%$ de los nódulos tiroideos operados corresponden a nódulos coloides y un $11 \%$ a $40 \%$ a adenomas foliculares $(7,33-35)$, sin embargo algunas series presentan al adenoma folicular como la lesión más frecuente $(18$, 36). El diagnóstico histológico de adenoma, tiene criterios precisos (25) y sensu strictu son lesiones monomórficas y monoclonales (37). La subclasificación histológica de los adenomas foliculares se ha visto que no tiene significación clínica (38).

En nuestro medio Rosell (39), de 1062 pacientes atendidos en el Servicio de Cirugía de Cabeza y Cuello del INEN, estudió 369 casos de adenomas, que se presentaron como nódulo solitario en el 94\%, no encontrando diferencia en cuanto a su frecuencia entre procedentes de ZDY y los de la costa. Los tipos más frecuentes fueron el simple (50.9\%), seguido del macrofolicular (23.6\%) y microfolicular (16.3\%).

El adenoma papilar es raro (12.39) y muchas veces puede corresponder en realidad a un carcinoma (12).

Hemos encontrado infiltrado linfocítico sólo en 6 casos (7.5\%), asociados a otras lesiones y sin otros hallazgos típicos de Tiroiditis de Hashimoto (TH). En la revisión hecha previamente en el servicio (23) sólo se halló 1 caso de 42, con esta patología. Durante el período de 1977-1986, de la totalidad de pacientes operados por patología tiroidea en el INEN y el Hospital Loayza, Venció H (40), sólo pudo encontrar 19 casos con cuadro histológico de Tiroiditis de Hashimoto que se presentaron como portadores de bocio uninodular. En la literatura la TH representa entre el 3.3\% a 17\% (33-35) de los casos de NT solitarios.

Hallamos un $32 \%$ de cáncer entre los pacientes operados, ligeramente mayor en los de la costa $32 \%$ vs $25 \%$, que es similar al $35.5 \%$ hallado por Cipriano (23) hace 18 años en una muestra menor, lo que indica la precisa selección clínica de los pacientes enviados a cirugía, aún antes del uso de la $\mathrm{ABC}$ en forma rutinaria. En nuestro medio Salem (41) ha encontrado un $20 \%$ de cáncer entre los pacientes operados por NT Frío en el INEN. En la literatura mundial el porcentaje varía entre $1.5 \%$ a $38.5 \%$ (13).

Se acepta que el mejor examen para el manejo del NT es la ABC, que ha permitido reducir en un $25 \%$ el número de intervenciones quirúrgicas innecesarias y duplicar el hallazgo de 
cáncer en los nódulos extraidos. Incluso su uso durante el acto quirúrgico puede ser más útil que la biopsia por congelación (42). Nuestra experiencia con la ABC de NT será materia de otra publicación.

Los factores clínicos de riesgo para cáncer de tiroides siguen teniendo valor para la selección de los pacientes para cirugía, como lo demuestra indirectamente nuestra serie, y su uso combinado con la $\mathrm{ABC}$ da mejores resultados, como lo señalan Hamming y col. (43), en una serie de 169 pacientes. La eficacia de la $\mathrm{ABC}$ fue de $92 \%$ en el grupo de alto riesgo clínico para cáncer y de $71 \%$ y $75 \%$ en los grupos de riesgo moderado y leve. En un reciente editorial Mazzaferri (44) recomienda que todo paciente con dos o más factores clínicos de riesgo para cáncer de tiroides y varones mayores de 60 años con NT frío deben ser operados cualquiera sea el resultado de la $\mathrm{ABC}$.

Con respecto al total de NT fríos encontramos un $13.5 \%$ de cáncer, cifra mayor al 5\% reportado en la literatura en series con más de 5,000 pacientes $(21,44)$. El porcentaje fue 1.6 veces mayor entre los de la costa $17.3 \%$ vs. $10.5 \%$.

En una de la series más grandes publicadas Belfiore y col (21) encuentran una prevalencia dos veces menor de cáncer entre pacientes con NT frío de ZDY en comparación con la zona control, $2.7 \%$ vs $5.3 \%$, sobretodo en mujeres. Entre los pacientes operados de ZDY el porcentaje de cáncer fue de $21.4 \%$ vs. $33.2 \%$ en la zona control (20), hallazgo similar al nuestro. Las mujeres de esta última región tenían más riesgo de ser portadoras de cáncer, que as de ZDY, así como también los pacientes de mayor edad de ambos sexos (21). Hubo una mayor frecuencia de cáncer folicular y anaplásico entre los pacientes de ZDY, cosa que no encontramos nosotros, quizá por el menor número de nuestra serie, por no haber incluido los pacientes que tenían NTF dentro de un bocio multinodular y por el hecho que estos pacientes son migrantes de ZDY con un tiempo de permanencia en Lima variable. En estudios poblacionales se observa que conforme aumenta el consumo de yodo en la dieta la proporción de cáncer papilar/folicular aumenta (45). En general el cáncer folicular de tiroides constituye entre el $25 \%$ a $40 \%$, de los cánceres de tiroides en pacientes que viven en ZDY (38).

En el $2.5 \%$ de nuestros pacientes había enfermedad de Graves concomitante, de los cuales ninguno fue operado debido a que la $\mathrm{ABC}$ no arrojó evidencia de malignidad y en otros por ser los nódulos quísticos, algunos de los cuales desaparecieron con la punción.

La presencia de Enfermedad de Graves con nódulo tiroideo frío que responde a la estimulación con TSH ha sido denominado Síndrome de Marine-Lenhart, por ser los primeros en describir el cuadro. Generalmente el nódulo es benigno y se presenta en $2.7 \%$ a 4.3\% de los casos de Enfermedad de Graves en zonas sin deficiencia de yodo (46).

Por otra parte la prevalencia de nódulos tiroideos en pacientes con Enfermedad de Graves, es mayor en ZDY (47) y se ha postulado que el cáncer tiroideo tiene un curso más agresivo en casos de hipertiroidismo $(48,49)$, aunque esto es debatido (50).

En conclusión hemos encontrado que el NTF es el tipo más frecuente de bocio nodular en precedentes de la costa mientras que el BMN lo es en migrantes de ZDY. La frecuencia de 
cáncer fue de $29 \%$ en la serie total. La menor frecuencia en procedentes de ZDY no fue significativa. Los pacientes de ZDY fueron de mayor edad al momento del diagnóstico.

\section{Agradecimiento:}

A los doctores Jaime Urbina, José Carrión y Hugo Arbañil por su colaboración en la revisión del archivo de Patología.

\section{Correspondencia:}

Dr. Jaime Villena

Instituto de Investigaciones de Altura. Apartado 6043. Lima 100, Perú.

\section{REFERENCIAS BIBLIOGRAFICAS}

1.Vander JB, Gaston EA, Dawber TR. The significance of Non-toxic Thyroid Nodules. Final Report of a 15-year Study of the Incidence of Thyroid Malignancy. Ann Intern Med 1968; 69:537.

2.Tumbridge WMG, Evered DC, Hall R, et al. The spectrum of thyroid disease in a community: The Whickham Survery. Clin Endocrinol 1977; 7:481.

3.Vigneri R. Studies in the goiter endemia in Sicily. J Endocrinol Invest 1987; 11:831.

4.Ville J. Aspectos Clínicos del Bocio Nodular. Experiencia de 15 años. Bol Soc Perú Med Interna 1992; 5: 71-76.

5.Robins J, Moderador. Thyroid Cancer: a letal endocrine neoplasm. Ann Intern Med. $1991 ; 115: 133-147$.

6.Ashcraft MW, Van Herle AJ. Managment of thyroid nodules. II: Scanning techniques, thyroid supressive therapy and fine needle aspiration. Head Neck Surgery 1981; 3:297-322.

7.Mazaferri EL. Managment of a Solitary Thyroid Nodule. N Eng J Med 1993; 328:533559.

8. Sheppard MC, Franklyn JA. Managment of the single thyroid nodule. Clinical Endocrinoly 1992; 37:398-401.

9.Ridgway ECH. Clinican. Evaluation of a Solitary Nodule J Clin Endocrinol Metab 1992; 74:231-235.

10.Grennspan F. The problem of the nodular goiter. Med Clin North Am 1991; 75:195-209.

11.Griffin JE. Management of Thyroid Nodules. Am J Med Sci 1988; 296:336-347.

12.Mazzaferri EL, de los Santos ET, Bofagha-Keyhani S. Solitary Thyroid Nodule: Diagnosis and Management. Med Clin North Am 1988; 72:1177-1208.

13.Rojeski MT, Gharib H. Nodular Thyroid Disease. Evaluation and Management. N Eng J Med 1985; 313:428-436.

14.Molitch ME, Beck JR, Dreisman M, Gottlier JE, Pauker SG. The Cold Thyroid Nodule: An Analysis of Diagnostic and Therapeutic Option. Endocrine Reviews 1984; 5:185-199.

15.Van Herle AJ, moderator. The thyroid nodule. Ann Intern Med 1982; 96:221-232.

16.Klonoff, Greenspan FS. The thyroid nodule. Advances in Internal Medicine 1982; 27:101-126.

17.Schttenfeld D, Gershman ST. Epidemiology of Thyroid Cancer. Cancer Journal for Clinicians 1978; 28:66-86. 
18.Cuello C, Correa P, Einsenberg H. Geographic Pathology of Thyroid Carcinoma. Cancer $1969 ; 23: 230-239$.

19. Whaner HW, Cuello C, Correa P, Uribe LF, Gaitan E. Thyroid Carcinoma in an Endemic Goiter Area, Cali, Colombia. Am J Med 1966;40:58-66.

20.Belfiore A, La Rosa G, Padova G, et al. The frecuency of Cold Thyroid Nodules and Thyroid malignacies in Patients from an Iodine Deficient Area. Cancer 1987; 60:30963102.

21.Belfiore A, La Rosa GL, Milazzo G, Lupo L, Regalbuto C, Vigneri R. Cancer Risk in Patients with Cold Thyroid Nodules: Relevante of Iodine Intake, Sex, Age and Multinodularity. Am J Med 1992; 93:363-369.

22.Pretell EA. Desórdenes por Deficiencia de Yodo (DDI). Generalidades. Situación en el Perú. En: Blanco de Alvarado Ortiz T, Gonzáles Mugaburu, eds. Situación Nutricional en el Perú. Lima: Propaceb, 1989:397.

23.Cipriani Enrique. Bocio Nodular Hiperfuncionante. Comentarios acerca de su etiopatogenia en una población migrante de zonas con bocio endémico. Tesis Doctoral. Lima, Perú. Universidad Peruana Cayetano Heredia. Programa Académico de Medicina, 1978.

24.Miller JM, Hamburger JI, Mellinger RC. The thyroid scintigram. II. The cold nodule. Radiology 1965; 85:702.

25.Sommers S. The Thyroid Gland. En: Bloodworth JMB Jr. Ed Endocrine Pathology:general and surgical. Baltimore: Williams \& Wilkins, 1982:155-203.

26.Benotti J, Benotti N. Protein Bound Iodine, total iodine and butanol extractable iodine by partial automation. Clin Chem 1963;9:408.

27.Wan MG. Determinación de Tiroxina por el método de unión competitiva por las proteínas. Tesis de Bachiller. Lima, Perú. Universidad Nacional Mayor de San Marcos, 1968.

28.Belfiore A, Sava L, Runello F, et al. Solitary Autonomously functioning Thyroid Nodules and iodine deficiency. J Clin Endocrinol Metab 1983; 56:283.

29.Villena J, Pretell EA. Bocio Nodular Tóxico en pacientes de la costa y de zonas yododeficientes. Revista Médica Herediana 1991.

30.De los Santos ET, Keyhani-Rofagha S, Cunningham J, Mazzaferri EL. Cystic Thyroid Nodules. The Dilemma of Malignant Lesions. Arch Intern Med 1990; 150:1422-1427.

31.Kuen Lee J, Fang-Ting T, Hong-Da L, Yi-Hong Ch, Kaplan MM, King-Nien Ching, Treatment if Recurrent Thyroid Cyst by Injection of Tetracycline or Minocycline. Arch Intern Med 1989; 149:599-601.

32.Edmonds CJ, Tellez M. Treatment of thyroid cysts by aspiration and injection of sclerosant. Br Med J 1987; 295:529.

33. Valimaki M, Vuorialho A, Bondestam S, Franssila K, Gylling H, Liewendahl K, Pamilo M, Sivula A, Soiva M. A clinicopathologic study of 233 patients with solitary thyroid nodule. En: Gordon, Gross \& Henneman eds. Progress in Thyroid Research. Rotterdam: Baikema, 1991:215-218.

34.Veith FJ, Brooks JR, Grigsby WP, Selenkow HA. The Nodular Thyroid Gland and Cancer. A Practical Approach to the Problem. New Engl J Med 1964; 270:431-36.

35.Werk EE, Vernon BM, Gonzáles JJ, Ungaro PC, McCoy RC. Cancer in Thyroid Nodules. A community Hospital Survey. Arch Intern Med 1984:144:474-76. 
36. Walfish PG, Hazani E, Strawbridge HTG, Murray M, Rosen IB. Combined Ultrasound and Needle Aspiration Cytology in the Assesment and Managment of Hypofunctioning Thyroid Nodule. Ann Intern Med 1977; 87:270-74.

37.A ebi U, Gerber H, Studer H. Thyroid Adenomas: A morphologically and functionally heterogeneous disease. En: Gordon, Gross \& Henneman eds. Progress in Thyroid Research. Rotterdam: Baikema, 1991:679-81.

38.Livolsi VA. Surgical Pathology of the Thyroid Gland. Major Problems in Pathology, Volumen 22. Philadelphia: WB Saunders Co 1990:173-212.

39.Rosell Ortiz Pío. Adenoma de la Glándula Tiroides en un país de Bocio Endémico. (Tesis de Bachiller). Lima-Perú. Universidad Peruana Cayetano Heredia. Programa Académico de Medicina, 1972.

40.Benzio Herschl. Tiroiditis de Hashimoto: Correlación clínico-patológica. (Tesis de Bachiller). Lima-Perú. Universidad Peruana Cayetano Heredia. Programa Académico de Medicina, 1987.

41.Salem Luis E. Cáncer de la glándula tiroides. Tesis Doctoral. Lima, Perú. Universidad Peruana Cayetano Heredia. Programa Académico de Medicina, 1973.

42.Hamburger J, Husain M. Contribution of Intraoperative Pathology Evaluation to Surgical Management of Thyroid Nodules. Clin Endocrinol Metab 1990; 19: 509-522.

43.Hamming JF, Goslings BM, van Steenis GJ, van Ravenswaay C, Hermans J, van de Velde CJH. The value of Fine-Needle Aspiration Biopsy in Patients with Nodular Thyroid Disease Divided Into Groups of Suspicion of Malignant Neoplasms on Clinical Grounds. Arch Intern Med 1990; 150: 113-116.

44.Mazzaferri E. Thyroid Cancer in Thyroid Nodules (Editorial): Finding a Needle in the Haystack. Am J Med 1992; 93:359-362.

45.Williams ED. Dietary Iodide and Thyroid Cancer. En: Hall R, Kobberling J (eds). Thyroid Disorders Associated with Iodine Deficiency and Excess. Serone Symposia Publications from Raven Press, 1985; Vol 22:201-207.

46.Park HM, Zieverink S, Ransburg RC, Yum MN, Maxon HR, UY Ryo. Marine-Lenhart Syndrome (Graves'disease with poorly functioning nodules). En: ME Stockigt ME, Nagataki S (eds). Thyroid Research VIII. Camberra, Australia Academy of Sciences, 1980. p:641-644.

47.Reinwein D, Benker G, Konig MP, Pinchera A, Schatz H, Schleusener A. The different types of hyperthyroidism in Europe. Result of a prospective survey of 924 patients. J Endocrinol Invest. 1988; 11:193-200.

48. Belfiore A, Garafalo MR, Giuffrida M, et al. Increased aggresiveness of thyroid cancer in patients with Graves's disease. J Clin Endocrinol Metab 1990; 70: 830.

49.Paul SJ, Sisson JC. Thyrotoxicosis caused by Thyroid Cancer. Clin Endocrinol Metab 1990; 19: 593.

50.Hales IB, McElduff A, Crummer P, Clifton-Bligh P, Delbridge L. Hoschl R y col. Does Graves'Disease or Thyrotoxicosis Affect the Prognosis of Thyroid Cancer. J Clin Endocrinol Metab 1992; 75:886-889. 